\title{
The Effect of Personality on Collaborative Task Performance and Interaction
}

\author{
Sinéad Mc Givney, Alan F. Smeaton and Hyowon Lee \\ CLARITY: Centre for Sensor Web Technologies and \\ Centre for Digital Video Processing, \\ Dublin City University, Glasnevin, Dublin 9, Ireland.
}

Summary. Collocated, multi-user technologies, which support group-work are becoming increasingly popular. Examples include MERL's Diamondtouch and Microsoft's Surface, both of which have evolved from research prototypes to commercial products. Many applications have been developed for such technologies which support the work and entertainment needs of small groups of people. None of these applications however, have been studied in terms of the interactions and performances of their users with regards to their personality. In this paper, we address this research gap by conducting a series of user studies involving dyads working on a number of multi-user applications on the DiamondTouch tabletop device.

\subsection{Introduction}

The personality composition of groups of people working collaboratively on shared tasks has been shown to be an important predictor of performance. For instance, a study of 63 virtual teams found that Extraversion was an important personality trait to promote group interaction and teams with lower variances in Extraversion levels did better [6. Collocated, touch-sensitive, groupware technologies, such as the DiamondTouch [7] and Microsoft's Surface as well as many new applications that support the work of small groups of people, such as photo management and spatial planning applications, are continually growing in sophistication. User studies on such applications and technologies thus far have failed to examine whether the combined personalities of small groups of people, working together on such collaborative technologies, have an impact on their performance and interaction. Here, we address this research gap by conducting a series of three detailed user experiments in order to analyse the effects of the combined personalities of dyad:11 on different collaborative application interface and task constraint variations. From these experiments, we can discover what personality traits significantly impact dyad performance and interaction.

\footnotetext{
${ }^{1}$ A dyad refers to a group containing two members
} 
This paper proceeds as follows. Section 1.2 provides an overview of previous research conducted with regards to the effects of group personality on task performance. In Section 1.3 we describe the systems that we designed for our user experiments. We follow this with a description of our experimental methodology in Section [1.4 In Section 1.5] we outline the results we obtained from our experimentation, in terms of the personality traits, if any, that affect the performance and interaction of dyads. Finally, we list our overall conclusions in Section 1.6

\subsection{Group Personality Studies}

Much research has been conducted to study the effect of the personality composition of groups on group performance. Rutherfoord [1], conducted a study with groups of people to determine whether those with a heterogeneous personality composition were more productive and enjoyed working together more than those with a homogenous personality composition. Twenty-two students participated in this study, which involved developing a game management system for an Athletic Association. Participants completed the Keirsey Temperament Sorter, which categorises personality along the Myers-Briggs scale 12 .

Results showed that the homogenous control groups experienced more problems on a personal level, rather than technical problems. The heterogeneous experimental groups conveyed a broader and more varied style of problem-solving and interacted more. They discussed about alternative solutions, devised more creative and effective ideas, worked together outside class hours more and were generally found to be stronger and more effective.

Gorla and Lam [5] distributed a questionnaire-based survey to 92 employees from 20 small software development teams (from three people to seven people in size), to determine what combinations of personalities resulted in the best-performing teams. The survey elicited information about the amount, quality, effectiveness and efficiency of the work the employees had done, as well as the frequency that their schedule and budget were adhered to. Participants' personalities were profiled using the Keirsey Temperament Sorter.

Results showed that team leaders with Intuitive, Feeling and Judging traits performed better. Heterogeneity of personality between the team leader and the team members, particularly in the Extravert/Introvert and Intuitive/Sensing dimensions, proved to be more successful, though heterogeneity among team members had no significant effect. Thinking type systems analysts performed better, as their roles incorporated more tasks than in a larger team. Extraverted programmers performed better than Introverted programmers. Diverse expertise and an appropriate means of sharing this information was also important.

Balthazard et al. [6] studied the performance of 63 virtual teams, composed of $248 \mathrm{MBA}$ professionals, with respect to each members' level of expertise and 
Extraversion, as well as the interaction style employed by the group. Participants completed a Five Factor Model profile and an online "Ethical Decision Challenge", first individually to determine each person's level of expertise, then as a randomly constructed group. Group members could communicate through an online chat and conference tool. When the task was completed, participants completed a Group Style Inventory ${ }^{\mathrm{TM}}$, which assessed interaction behaviours within the group, and a group process questionnaire, which assessed process satisfaction and "buy-in" into the consensus solution.

Analysis of the results showed that team performance was best predicted by expertise in the group. In general, it was mostly the interaction style of the groups that had predictive power on the contextual outcomes in virtual environments. Extraversion was found to be an important trait to promote group interaction and teams with lower variance in extraversion levels did better.

A study was conducted in 2] into online communities and the factors that promote participation in these groups. The online community, consisting of eight groups in the study, used a movie recommender system. Uniqueness and similarity combinations were tested to see which condition resulted in the largest participant contribution. Four of the groups were sent a weekly e-mail informing them of the unique perspective they could bring to the current discussion group. The remaining four groups acted as control groups. Groups were defined as "similar" if they typically watched the same movies and agreed on their reviews of these movies. Dissimilar groups either just watched different movies, or disagreed on movies they had watched. Again, there were four similar groups and four dissimilar groups.

Results showed that dissimilar groups that were supplied with uniqueness information contributed more to these online communities. These results were contrary to the authors' hypothesised results and the many theories and studies completed in social psychology e.g. 3], in that dissimilar groups participated more than similar groups and this diversity was significant.

In the studies we carried out and report on, we were not only interested in the effects of dyad personality composition on their performance and interaction styles, but also the effects of the personality traits exhibited by the application interface they used. In the following section, we describe the systems we used for our user-studies.

\subsection{Systems Used}

Here, we provide an outline of the technology that we developed our systems on, followed by an outline of three collaborative systems that we designed and used. 


\subsubsection{DiamondTouch and DiamondSpin}

The DiamondTouch is a multi-user, tabletop device designed and developed by researchers at the Mitsubishi Electric Research Labs (MERL) in Boston, Mass. The tabletop's surface is touch-sensitive and the technology can uniquely distinguish the touchpoints of up to four users. This is enabled by placing signal receiver mats under each user and connecting these mats to the underside of tabletop (transmitter). Once the user touches the tabletop's surface, they complete a circuit and are capacitively coupled with the tabletop. A more detailed description of the DiamondTouch can be found in [7.

DiamondSpin 8 is a Java-based Software Development Toolkit, also designed by researchers and collaborators at MERL. The toolkit contains a polar to Cartesian transformation engine, which handles the rotation and orientation of objects displayed on the DiamondTouch's surface. The toolkit has a well-defined API containing 30 Java classes and interfaces. It also uses pure JAVA 2D with JAI (Java Advanced Imaging) and JMF (Java Media Framework).

We now describe three collaborative system variants.

\section{Memory Game}

Memory Game is a competitive card game where players try to find matching pairs of cards. For our version, 24 cards are displayed face-down on the tabletop. Each player overturns two cards when it is their turn and if these match, then that player is given one point and another turn. If the cards do not match, control passes to the other player. Scores are displayed at the side of the interface and oriented to each player. The player with the highest number of matching pairs wins the game.

We made this game collaborative by requiring dyads to find pairs together. This game commenced when the first player touched the tabletop screen (Player 1). This player was then required to overturn the first card in this and in subsequent games. Player 2 then had to select a card that they believed matched the already overturned card. Their choice of card was predominantly the result of both players discussing options and sharing potential match location information. We used 4 different sets of cards in this system.

Two different rules or constraints were imposed, the first of which was to find all matching pairs accurately while incurring as few mismatched pairs as possible. The second rule required that users find all matches as quickly as possible, regardless of the number of mismatched pairs they incurred. After the second game was played on each rule, players were asked to switch sides, so that each got a turn at being match-chooser and match-finder for each rule imposed for half the task, making it fairer. Figure 1.1 illustrates the Accuracy Memory interface. 


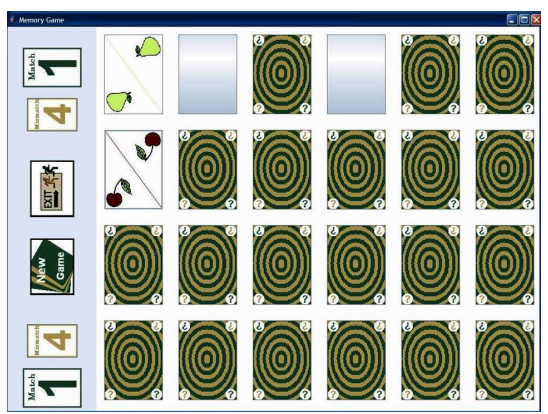

Fig. 1.1. Accuracy Memory Game interface

\subsubsection{Físchlár-DT}

Físchlár-DT is a two-person video search system that our research group built on the DiamondTouch for the annual TRECVid (Text Retrieval Conference for Video) 2005 workshop [10]. TRECVid is a benchmarking conference series, where participants compare video retrieval and analysis techniques on a large shared test dataset [13.

The aim of this search task was to find as many video shots (sections of video) as possible out of a supplied repository, that were relevant to a given multimedia topic using a video search system e.g. "Find shots of Tony Blair'. Each shot was represented by an image called a keyframe.

Físchlár-DT was developed using the DiamondSpin SDK, to easily handle the rotation and orientation of objects on the interface. One interface we developed for these experiments had a number of hot-spots which enabled the user to carry out specific actions. Each hot-spot had an associated distinctive sound, which both made their partner aware that a certain function had been invoked and also provided feedback to the user that it was invoked properly. Users could type a text query (using a pop-up keyboard) into a movable search box located in the bottom right-hand corner of the screen. The "search" button then delivered up to 20 keyframes displayed around the table with more relevant keyframes displayed closer to the centre of the screen. This is illustrated in Figure 1.2

Dragging a shot keyframe over the "Play" hot-spot commenced playback of the shot on an external monitor. The "Browse" hot-spot displayed the next ten and previous ten shots in that particular news broadcast, to the shot selected. The "Find Similar" hot-spot displayed keyframes from 20 shots that were similar to the selected shot keyframe, by comparing MPEG-7 descriptors of that keyframe to the rest of the keyframes in the collection. "Remove" deleted the selected keyframe from the screen, not to be retrieved again for that particular search task. Finally, if a keyframe was moved into the "Saved Area", the shot was marked relevant with a yellow border and stamp. Any or 


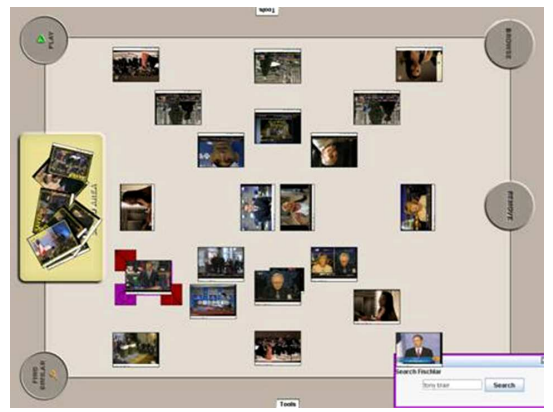

Fig. 1.2. Fischlár-DT: Awareness interface

all of these functions could be invoked by either user by dragging a keyframe onto the appropriate hot-spot.

Similar to the Memory Game system, one of our user studies involving the Físchlár-DT system imposed different rules on the participating dyads. The first constraint imposed in our our first search-based task (Físchlár-DT 1) was a ten minute time-limit, where dyads had to find as many relevant shots as possible to two specified topics - "Find shots of Condoleeza Rice" and "Find shots of people shaking hands". The order of these topics was switched from dyad to dyad to avoid order bias. The other rule demanded that dyads find ten shots in total, that were relevant to two topics (i.e. "Find shots of tanks or other military vehicles" and "Find shots of banners or signs"). The topics used were a subset of the topics used for the TRECVid 2005 interactive video retrieval experiments, since the relevance judgements were known. By this, we mean that we had lists of shots that were deemed relevant to each topic by manual assessors as part of TRECVid.

For our final user experiment, Físchlár-DT 2, we altered the interface to the Físchlár-DT system, to give two variations that exploited the Extraversion personality trait. Figure 1.3 shows the Extravert interface. Here, we chose bright, highly saturated colours, boxes and sharp edges [14. We removed the "Find Similar" hot-spot and instead, when a user saved a keyframe, the system displayed four keyframes representing shots it thought were similar to that saved. These were then displayed in a "Suggested Clips" area, located at the centre of the table. This was in keeping with the idea that system-initiated interaction is preferred by extraverted people [4].

The "Browse" hot-spot was moved from the top right-hand corner of the table, to the bottom left-hand corner where the "Find Similar" hot-spot was originally located, hence making the interface more balanced.

For our Introvert interface (see Figure 1.3), we used softer, pastel colours. The hot-spots were round in shape, the text was also more rounded and we moved the saved area to the centre of the table [14. Each of the functions were duplicated for each user, allowing them to work more independently 


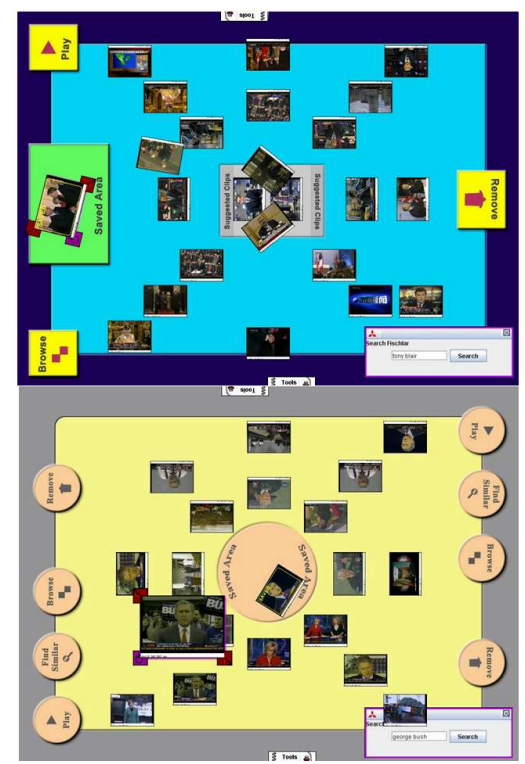

Fig. 1.3. Extravert and Introvert Físchlár-DT 2 Interfaces

and quietly, which was in keeping with the introverted personality type. This however, did result in users being less aware of each others' actions. The "Find Similar" hot-spot was brought back into this version.

Dyads searched for 3 topics on each of these interfaces, totaling 6 topics altogether for the entire session. These again, were a subset of the TRECVid 2005 topics. There was a 5-minute limit imposed on dyads for each topic to find as many shots as possible that were relevant to that topic. The order of presentation of the interfaces was changed for each dyad.

\subsection{Experimental Methodology}

Here, we give an overview of how we conducted our user experiments, from how our experiment participants were recruited to the experimental procedure we followed. We also outline how the participants' personalities were profiled.

\subsubsection{Participant Recruitment}

Participants were recruited from the general university student population via email. This email requested the participation of pairs of users, whom if selected, would be financially rewarded in return for completing our userstudies. 
We used the Five Factor model of personality to profile the personalities of our participants, which is the most widely accepted model of personality in the personality psychology community. This model describes the personality of individuals in terms of five personality traits i.e. Openness to Experience, Conscientiousness, Extraversion, Agreeableness and Neuroticism - OCEAN 11. The strength of these traits present in an individual are measured based on the responses of the individual to a specially designed personality questionnaire. Analysing these answers enables a percentage to be calculated for each trait e.g. $35 \%$ Extravert.

In this study, we believed that Extraversion would have the greatest impact on both the performance and the interaction of the group. Hence, when people initially responded to the recruitment email, we requested them to answer 6 short questions from the IPIP-Neo online personality questionnaire [15] which appeared to prevalently measure the Extraversion trait. After the first experiment, participants were asked to complete the short version (i.e. 60 questions) of an online IPIP-Neo personality questionnaire. 36 people in total took part in the studies, with just three females. Other females who had responded to the recruitment email, failed to show up at the experiments. There were no female/female dyads.

There was at least one week between each of the tasks, with each one lasting between 30 minutes and one hour. Before starting each task, dyads were introduced to the system interface variations or rule variations, as well as completing some pre-task training. Each participant also completed pre and post study questionnaires which elicited information about their age, background, prior experience and their opinions on the task they had just completed.

Footage of the user studies was captured using a CCTV camera, placed at a height above the tabletop. We subsequently annotated the interaction of dyads from this footage. We logged and time-stamped four types of communication requests (which could be verbal, gestural or both), responses (which could be verbal, gestural or both), comments and coordination errors. A coordination error was an action that one user took that interrupted their partner's work e.g. invoking an action without warning, that entirely changed the display. The recording of the studies was approved by our University's Ethics committee. In total, we recorded approximately 55 hours of video footage.

\subsubsection{Data Gathered}

We gathered a substantial amount of data in carrying out our user studies, which we categorised under two main headings:

\section{Explicitly Supplied User Data}

1. Questionnaire responses from users (both pre and post-study questionnaires), which elicited information regarding participant's age, course, familiarity with web searching and the DiamondTouch, frequency of working 
in groups, as well as their opinions on the systems used (i.e. ease of use, opinion of interface colours and layout etc.).

2. Personality Questionnaire responses (i.e. users' scores for the Big Five personality traits).

\section{System Recorded Data:}

3. Performance Data (i.e. user scores in the game experiments and the number of relevant shots saved in the search tasks).

4. User touchpoints on the tabletop (meaning exactly where on-screen each user touched).

5. CCTV footage of the user experiments and associated annotations.

Our analysis of this data involved identifying relationships between each of these data sources to study the effects of personality traits on performances and interactions on our collaborative tasks. We explain this in the next section.

\subsection{Results}

\subsubsection{Personality and Performance}

In order to explore whether correlations exist between the personalities of dyads and their performance, it was necessary to combine the trait scores of both dyad members. For the Extraversion trait, we tested a number of orderings, including a metric which we called "E-Dist". This measured the absolute difference between the Extraversion scores of both dyad members, as reported on their completed personality questionnaires. The idea here was that the closer each person's level of Extraversion was to that of their partner, the more similar and compatible they would be [3]. Hence, we anticipated a correlation between low E-Dist values and high levels of performance.

Our other metrics included "Avg. Extra", "Most Extra" member and "Most Intro" member. For "Avg. Extra", we simply averaged both dyad member's scores for the Extraversion trait. In the case of the "Most Extra" measure, we examined the Extraversion results for each dyad member and chose the more extravert member's result. Similarly, we noted the member of each dyad who had the lowest percentage of Extraversion for "Most Intro". We applied the same combination schemes to the other four personality traits (Openness to Experience (O-Dist, Avg. Open, Most Open, Least Open), Conscientiousness (C-Dist, Avg. Cons, Most Cons, Least Cons), Agreeableness (A-Dist, Avg. Agree, Most Agree, Least Agree) and Neuroticism (N-Dist, Avg. Neur, Most Neur, Least Neur)).

We used the Spearman rank correlation method (a special, non-parametric case of the Pearson product-moment rank correlation) to identify statistical relationships between the orderings of each of our personality trait combination metrics and ordering of dyad performance, which we decided was the most appropriate metric given the characteristics of our dataset. Table [1.1] 
displays the statistically significant relationships between dyad personality traits and performance (given a critical value of \pm 0.476 at $\alpha=0.05$ for a two-tailed test). Column 1 lists the tasks studied; column 2 lists the personality trait combination schemes that had a statistically significant effect on dyad performance and column 3 gives the respective correlation coefficients $\left(\mathrm{r}_{s}\right)$ calculated from our Spearman rank correlations. We note here that the combined dyad Extraversion and Neuroticism trait did not affect performance on any of our tasks - a surprising result, given the social nature of the tasks.

\begin{tabular}{|l|c|c|}
\hline Task & Related Traits & $\mathbf{r}_{s}$ value \\
\hline Accuracy Memory & Least Cons & -0.53 \\
& Least Agree & -0.53 \\
& Avg. Agree & -0.53 \\
\hline Speed Memory & - & - \\
\hline Fís-DT 1 (10 min) & O-Dist & -0.49 \\
\hline Fís-DT 1 (Find 10) & - & - \\
\hline Fís-DT 2 (Int) & Least Open & -0.54 \\
& Least Consc. & -0.47 \\
\hline Fís-DT 2 (Ext) & O-Dist & -0.64 \\
& Most Open. & -0.47 \\
\hline
\end{tabular}

Table 1.1. Correlations between performance and dyad personality traits

It is interesting to note that none of the personality traits affected performance on either of the 2 systems that required users to complete the task quickly, namely Speed Memory and the Físchlár-DT 1 (Find 10) system both of which required users to complete their respective tasks as quickly as possible. In terms of Físchlár-DT 1 (10 minute), and both Físchlár-DT 2 interfaces, we see that Openness to Experience is an important trait in relation to performance, though the correlations calculated indicate that dyads containing at least one person with a much lower Openness to Experience score than their partner perform better.

The strong negative correlation along the Least Cons combination scheme for Físchlár-DT 2 (Introvert interface) is also a surprising finding, since we believed that this system required both users to think before they acted. Since the functions were duplicated on this interface, there was more potential for users to interrupt their partner's work e.g. playing over each others' videos.

Both Conscientiousness and Agreeableness were important in the Accuracy Memory system where the Least Cons, Least Agree and Avg. Agree trait combination schemes all produced negative correlations. This implies that low Conscientiousness and low Agreeableness produced lower performances (the performance ranks of Accuracy Memory differed since a lower figure for performance indicated better performance/fewer mismatches). These resulting correlations were much more intuitive, since it would be important that dyad 
members were both conscientious (i.e. they lacked impulsiveness and thought before they acted) and agreeable, so that they made the fewest errors possible.

\subsubsection{Personality and Interaction}

We conducted a similar type of analysis for our interaction data for all dyads and all systems - that being the touchpoints recorded and the interactions annotated from the CCTV footage of the experiments. Again, we used the personality trait combination metrics that we listed in the previous subsection. Since the length of some of the tasks differed (i.e. Accuracy Memory, Speed Memory and Físchlár-DT 1 (Find 10)), we had to normalise the number of interactions per dyad so that they could be fairly compared. Hence, we took one minute of time as our normalising unit.

We look first at our touchpoint data. From Table 1.2 we see that Conscientiousness and Extraversion have a significant effect on the number of touchpoints for both Collaborative Memory game rules. This indicates that dyad members, whose level of Conscientiousness was similar (i.e. a low $C$ Dist), had fewer touchpoints per minute than those who had very different levels when working on Accuracy Memory. However, for Speed Memory, the $\mathrm{r}_{s}$ values obtained implied that dyads who had a high average Conscientiousness or where both members had high levels of Conscientiousness had fewer touchpoints. This was a logical finding, since a lack of impulsiveness and a more thoughtful approach to the game would be important here.

We also see that the Neuroticism personality trait is significantly correlated to the number of touchpoints in Speed Memory, where dyads who have similar levels of Neuroticism have fewer touchpoints. In addition, dyads containing at least one member with a high level of Neuroticism had significantly more touchpoints than those dyads whose members had lower levels. Neuroticism also has a significant negative correlation on Físchlár-DT 1 along the $N$-Dist metric i.e. dyads whose members' level of Neuroticism was similar had more touchpoints. Openness to Experience produced a negative correlation on Físchlár-DT 2 (Introvert Interface) along the Most Open metric i.e. dyads where at least one dyad member had a relatively high level of openness had fewer touchpoints.

Finally, on Físchlár-DT 2 (Extravert interface), negative correlations were calculated for the Most Agree and A-Dist metrics. These results imply that dyads with at least one more agreeable member had fewer touchpoints, while dyads whose members had more similar levels of Agreeableness had more touchpoints.

Finally, we look at the annotations (interactions) that we made from the CCTV recordings of the experiments. Due to the fact recordings were corrupted for some dyads in some systems, our sample sizes and subsequently our critical values were different for each system. For both Collaborative Memory systems and both Físchlár-DT 1 rules, our sample size was $\mathrm{n}=17$. With an $\alpha$ of 0.05 (two-tailed test), our critical value here was 0.507 . Our sample size 


\begin{tabular}{|l|c|c|}
\hline & Related Traits & $\mathbf{r}_{s}$ value \\
\hline Accuracy Memory & C-Dist & 0.49 \\
\hline Speed Memory & Avg. Cons & -0.62, \\
& Least Cons & 0.48 \\
& N-Dist & 0.53 \\
& Most Neur & 0.5 \\
\hline Fís-DT 1 & N-Dist & -0.59 \\
\hline Fís-DT 2 (Int) & Most Open & -0.49 \\
\hline Fís-DT 2 (Ext) & Most Agree & -0.51 \\
& A-Dist & -0.48 \\
\hline
\end{tabular}

Table 1.2. Traits significantly related to touchpoints for each system and associated combination metric.

for Físchlár-DT 2 Extravert interface was 16, with a critical value of 0.507 . Lastly, our Físchlár-DT 2 Introvert system had an $\mathrm{n}$ of 15 and a critical value of 0.545 .

\begin{tabular}{|l|c|c|}
\hline & Related Traits & $\mathbf{r}_{s}$ value \\
\hline Accuracy Memory & $\begin{array}{c}\text { Avg. Extra } \\
\text { Most Intro }\end{array}$ & 0.63 \\
& - & 0.63 \\
\hline Speed Memory & - & - \\
\hline Fís-DT 1 (10 min) & Avg. Extra & 0.51 \\
\hline Fís-DT 1 (Find 10) & Avg. Extra & 0.56 \\
\hline Fís-DT 2 (Int) & Least Neur & -0.59 \\
& Avg. Extra. & 0.70 \\
\hline Fís-DT 2 (Ext) & Avg. Agree & 0.55 \\
& Most Extra. & 0.74 \\
\hline
\end{tabular}

Table 1.3. Traits significantly related to interactions and associated combination metric for each system.

It is clear from Table 1.3 that the strong significant positive correlation between the Avg. Extra metric and interactions indicates that users with higher average Extraversion values have a greater number of interactions (communication) on the Accuracy Memory game and Físchlár-DT 1 (both rules) and Físchlár-DT 2 (Introvert Interface). Físchlár-DT 2 (Extravert) shows a highly significant correlation between Most Extra and interactions, indicating that dyads with at least one highly extraverted member communicated more. This would appear to support previous psychological research showing that people with high levels of Extraversion are talkative and sociable.

The Most Intro metric, which also showed a significant positive $\mathrm{r}_{s}$ value of 0.63 for the Accuracy Memory system, indicates that those dyads that contained at least one very introvert member were less likely to communicate than those with more extraverted members. Físchlár-DT 2 (Introvert interface) dis- 
plays a significant negative correlation alog the Neuroticism personality trait i.e. dyads where both members were highly neurotic had fewer interactions. Físchlár-DT 2 (Extravert interface) showed a significant, positive correlation between increasing Avg. Agree and increasing interactions i.e. dyads whose members were on average more agreeable, had more interactions. These were intuitive findings.

\subsection{Conclusions}

From the results presented above, we can see that the combination of dyad members' personalities does impact their performance and interaction. However, the personality traits that most prevalently have an impact differ from task to task. One very obvious trend was that Extraversion had an impact on tasks that did not enforce very short time constraints. Openness to Experience had an impact on performance on search tasks, while Agreeableness was important for tasks where tight collaboration was required (in our case, the Accuracy Memory system). Conscientiousness was also found to have an impact on performance, though there was no apparent trend across the systems it impacted. All personality traits aside from Extraversion had some impact on the number of touchpoints that each dyad incurred. Conscientiousness impacted the number of touchpoints incurred on both our collaborative Memory Game systems. However, there were no other obvious trends concerning trait impact on touchpoints and interface or task type.

Collaborative tasks, such as tagging on the net, both for work and for leisure pursuits, are growing and becoming more widespread through the use of devices like the DiamondTouch and Microsoft's Surface. From our rather mixed results we can conclude that as this growth happens, system designers do need to be made aware of findings such as ours as they will influence, in one way or another, the effectiveness of systems built to support collaboration.

Interesting future experiments may look at a comparison of the performance of one person executing these tasks against the performance of dyads working on such a collaborative technology; or determining whether similar trends can be observed when users are collaboratively working together in a distributed/virtual environment.

\subsection{Acknowledgements}

The authors gratefully acknowledge the support of Mitsubishi Electric Research Labs. Thanks also to Emilie Garnaud for the use of original Memory Game system. This work is supported by the Irish Research Centre for Science, Engineering and Technology (IRCSET) and Science Foundation Ireland under grant numbers 03/IN.3/I361 and 07/CE/I1147. 


\section{References}

1. Rutherfoord, R.H. : Using personality inventories to form teams for class projects: a case study. In: SIGITE '06, pp 9-14. ACM, Minnesota, USA (2006)

2. Ludford, P.J., Cosley, D., Frankowski, D. and Terveen, L. : Think different: increasing online community participation using uniqueness and group dissimilarity. In CHI '04, pp 631-638. ACM, Vienna, Austria (2004)

3. Byrne, D. and Nelson, D. : Attraction as a Linear Function of Proportion of Positive Reinforcements. Journal of personality and social psychology (4), pp = 240-243, American Psychological Association (1965)

4. Reeves, B., Nass, C.: The Media Equation. Chapter on Media and Personality, page 75. CSLI Publications (1996)

5. Gorla, N., Lam, Y.W.: Who should work with whom?: building effective software project teams. In: Communications of the ACM, 47, pp 79-82. ACM, NY, USA (2004)

6. Balthazard, P., Potter, R. E., Warren, J.: Expertise, extraversion and group interaction styles as performance indicators in virtual teams: how do perceptions of IT's performance get formed? In: ACM SIGMIS Database, 35, 41-226. ACM, NY, USA (2004)

7. Dietz, P., Leigh, D.: DiamondTouch: a multi-user touch technology. In: UIST '01, pp. 219-226. ACM, Orlando, Florida (2001)

8. Shen, C., Vernier, F. D., Forlines, C., Ringel, M.: DiamondSpin: an extensible toolkit for around-the-table interaction. In: CHI '04, pp. 167-174. ACM, Vienna, Austria (2004)

9. Pickford, R.W.: Psychology and Visual Aesthetics. Hutchinson Educational LTD, London (1975)

10. Foley, C., Gurrin, C., Jones, G., Lee, H., Mc Givney, S., OConnor, N., Sav, S., Smeaton, A.F., Wilkins, P.: TRECVID 2005 Experiments at Dublin City University. In: TRECVID 2005: - Text REtrieval Conference TRECVID Workshop. Gaithersburg, Maryland (2005)

11. Costa, P.T., McCrea, R.R.: Revised NEO Personality Inventory (NEO PI-R) and NEO Five-Factor Inventory (NEO-FFI). Psychological Assessment Resources (1937)

12. Moad, J.: Psych Tests for MIS Staff: Is This Nuts?. Datamation (1994)

13. Smeaton, A., Over, P., Kraaij, W.: Evaluation campaigns and TRECVid. In: MIR '06, pp. 321-330. ACM, Santa Barbara, California, USA (2006)

14. Karsvall, A.: Personality preferences in graphical interface design. NordiCHI '02, pp. 217-218. ACM, Aarhus, Denmark (2002)

15. Short Form for the IPIP-Neo (International Personality Item Pool Representation of the NEO PI-R), http://www.personal.psu.edu /faculty/j/5/j5j/IPIP/ipipneo120.htm 